\title{
Development of negative muonium ion source for muon acceleration
}

\author{
R. Kitamura $\odot,{ }^{1,}$ S. Bae, ${ }^{2}$ S. Choi $\odot,{ }^{2}$ Y. Fukao, ${ }^{3}$ H. Iinuma, ${ }^{4}$ K. Ishida,${ }^{5}$ N. Kawamura $\odot,{ }^{3}$ \\ B. Kim $\odot,{ }^{2}$ Y. Kondo, ${ }^{1,4}$ T. Mibe $\odot,{ }^{3}$ Y. Miyake, ${ }^{3}$ M. Otani, ${ }^{3}$ G. P. Razuvaev, ${ }^{6,7,8}$ \\ N. Saito ${ }^{9},{ }^{9}$ K. Shimomura, ${ }^{3}$ and P. Strasser ${ }^{3}$ \\ ${ }^{1}$ Japan Atomic Energy Agency (JAEA), Tokai, Naka, Ibaraki 319-1195, Japan \\ ${ }^{2}$ Department of Physics and Astronomy, Seoul National University, Seoul 08826, Republic of Korea \\ ${ }^{3}$ High Energy Accelerator Research Organization (KEK), Tsukuba, Ibaraki 305-0801, Japan \\ ${ }^{4}$ Ibaraki Univ., Mito, Ibaraki 310-8512, Japan \\ ${ }^{5}$ RIKEN, Nishina Center, Wako, Saitama 351-0198, Japan \\ ${ }^{6}$ Budker Institute of Nuclear Physics SB RAS, Novosibirsk 630090, Russia \\ ${ }^{7}$ Novosibirsk State University, Novosibirsk 630090, Russia \\ ${ }^{8}$ Pulkovo Observatory, St. Petersburg, 196140, Russia \\ ${ }^{9} \mathrm{~J}-\mathrm{PARC}$ center, Tokai, Naka, Ibaraki, 319-1195, Japan
}

(Received 24 June 2020; revised 29 January 2021; accepted 19 February 2021; published 24 March 2021)

A negative muonium ion $\left(\mathrm{Mu}^{-}\right)$source using an aluminum foil target (Al target) was developed as a low-energy muon source. $\mathrm{Mu}^{-}$ions are produced by irradiating the $\mathrm{Al}$ target with a 3-MeV positive muon $\left(\mu^{+}\right)$beam and observed using a microchannel plate. An experiment to produce $\mathrm{Mu}^{-}$ions was conducted to evaluate the performance of this $\mathrm{Mu}^{-}$ion source. The measured event rate of $\mathrm{Mu}^{-}$ions was $(1.7 \pm 0.3) \times 10^{-3} \mathrm{Mu}^{-} / \mathrm{s}$ when the event rate of the incident $\mu^{+}$beam was $1.3 \times 10^{6} / \mathrm{s}$. The experiment was conducted at the Muon Science Establishment, D-line in the Materials and Life Science Experimental Facility within the Japan Proton Accelerator Research Complex. The formation probability, defined as the ratio of the $\mathrm{Mu}^{-}$ions to the incident muons on the Al target, was $\left(1.1 \pm 0.2(\text { stat })_{+0.1}^{-0.0}\right.$ (syst $\left.)\right) \times 10^{-6}$. This $\mathrm{Mu}^{-}$ion source was first adopted in the commissioning of the muon accelerator at the D-line, and the event rate of the accelerated $\mathrm{Mu}^{-}$ions was consistent with the expectation. $\mathrm{This}_{\mathrm{Mu}}^{-}$ion source boosted the development of the muon accelerator, and the practicality of this low-energy muon source obtained using a relatively simple apparatus was demonstrated.

DOI: 10.1103/PhysRevAccelBeams.24.033403

\section{INTRODUCTION}

A negative muonium ion $\left(\mathrm{Mu}^{-} ; \mu^{+} e^{-} e^{-}\right)[1-3]$ is the bound state of one positive muon $\left(\mu^{+}\right)$and two electrons $\left(e^{-}\right)$and is known to be produced in a vacuum by irradiating an aluminum foil target (Al target) with a $\mu^{+}$ beam. $\mathrm{Mu}^{-}$ion production has primarily been studied in the context of atomic physics, for example, as it pertains to the Lamb shift because $\mathrm{Mu}^{-}$ions are analogous to negative hydrogen ions. In this experimental study, we focus on the fact that $\mathrm{Mu}^{-}$ions are emitted with a small kinetic energy of approximately $O\left(10^{-1}\right) \mathrm{keV}$ and propose a new usage of $\mathrm{Mu}^{-}$ions as a method to cool muon beams.

Typically, muon beams produced with accelerators have been used to research particle physics and in studies

\footnotetext{
"rkita@post.j-parc.jp
}

Published by the American Physical Society under the terms of the Creative Commons Attribution 4.0 International license. Further distribution of this work must maintain attribution to the author(s) and the published article's title, journal citation, and DOI. regarding materials and life science, for example, using the muon spin rotation, relaxation, and resonance $(\mu \mathrm{SR})$ method [4]. However, since muons are produced as tertiary beams via the decay of pions originating from the primary proton beam reacting with the target nuclei, the emittance of muon beams is intrinsically large. A novel muon beam with a small emittance has been long sought-after to achieve a precision measurement of the muon dipole moment in particle physics [5]. Moreover, such a beam has potential use in research regarding the new phenomena around the interface of the sample for materials and life science $[6,7]$.

In the precision measurement of the muon dipole moment project in the Japan Proton Accelerator Research Complex (J-PARC E34), a muon beam with $p_{t} / p_{l}<10^{-5}$, where $p_{l}$ is the longitudinal momentum, and $p_{t}$ is the transverse momentum, is required to reduce systematic uncertainties when using a new method. This unique muon beam is obtained by producing ultra-slow muons (USMs) with a mean energy of $0.02 \mathrm{eV}$ from the surface muon beam (which is produced from the pion decay stopped around the surface of the muon production target) and then reaccelerating the 
USMs using a linac. We call this muon beam a reaccelerated thermal muon beam. The laser-resonant ionization of the muonium atom $\left(\mathrm{Mu} ; \mu^{+} e^{-}\right)[8-10]$ was developed to obtain USMs. To obtain slow muons, several methods like socalled moderator method [1,11-13] were often utilized. However, the laser-ionization method is adopted due to the synthesis of the J-PARC pulsed muon beam.

A reaccelerated thermal muon beam requires both the beam cooling, like that caused by an USM source, and a muon accelerator [14-19]. These two important techniques should be developed individually in a timely manner for the early realization. However, to develop a muon accelerator, muons with low energy are required; with exception of USM source, few low-energy muon sources exist. Therefore, in this study, a $\mathrm{Mu}^{-}$ion source using an $\mathrm{Al}$ target is investigated as a pseudo-USM source. For example, since a radio-frequency quadrupole (RFQ) linac, which is applied to the initial accelerator of the muon linac $[16,20]$, requires an input energy of $5.6 \mathrm{keV}$, the $\mathrm{Mu}^{-}$ ion source, the energy of which is less than $1 \mathrm{keV}$, is suitable for the RFQ after electrostatic preacceleration. Although the formation probability of the $\mathrm{Mu}^{-}$ion source is smaller than that of USMs, and its transverse emittance is larger $\left(O\left(10^{-3}\right)\right.$ and approximately $1 \pi \mathrm{mm}$ mrad, respectively, for USMs) [5], the $\mathrm{Mu}^{-}$ion source is highly superior in terms of easy handleability.

To apply the $\mathrm{Mu}^{-}$ion source to the muon acceleration, the formation probability of the $\mathrm{Mu}^{-}$ion is quite important to estimate the beam transmission efficiency. The precursor study [2] inspires us to apply the $\mathrm{Mu}^{-}$ion emission from metal surfaces to the ion source of the accelerator. To realize this in the practical experimental apparatus, the detailed understanding is necessary concerning the $\mathrm{Mu}^{-}$ ion emission rate, the energy, and the emission angle distribution. To conduct the commissioning of the muon accelerator in a realistic period, the emission rate of $\mathrm{Mu}^{-}$ ions was needed to be $O(1) \mathrm{Mu}^{-} / \mathrm{s}$ on the rear surface of the $\mathrm{Al}$ target. In this study, the event rate of the produced $\mathrm{Mu}^{-}$ion beam was measured and the result was compared with the Monte-Carlo simulation where the practical condition from the incident muon beam to the detector was reproduced. It is the essential information to evaluate the beam transmission in the commissioning of the muon accelerator using our $\mathrm{Mu}^{-}$ion source. In this paper, first, the experimental setup is described in Sec. II. Next, the simulation is described in Sec. III, and the experimental results are described in Sec. IV. The measured formation probability of $\mathrm{Mu}^{-}$ions is discussed in Sec. V, and the conclusion is presented in Sec. VI.

\section{EXPERIMENTAL SETUP}

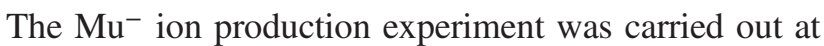
the muon experimental facility known as the Muon Science Establishment (MUSE), D-line in the J-PARC Materials and Life Science Experimental Facility [7]. The event rate of the incident $\mu^{+}$beam in this experiment was $1.3 \times 10^{6} / \mathrm{s}$ $\left(5.2 \times 10^{4} /\right.$ pulse with a repetition rate of $\left.25 \mathrm{~Hz}\right)$. The momentum of the incident $\mu^{+}$beam was set to $25 \mathrm{MeV} / c$ to maximize the number of the $\mu^{+}$'s stopped at the downstream surface of the Al target. The momentum spread of the D-line was $4 \%$ in RMS (root-mean-square).

Figures 1 and 2 are schematic drawings; the former shows the experimental apparatus, and the latter shows the $\mathrm{Al}$ target and an electrostatic acceleration and focusing system called the Soa lens [21]. The incident $\mu^{+}$beam passes through a stainless steel window (SUS window) with a thickness of $50 \mu \mathrm{m}$, which separates the vacuum of

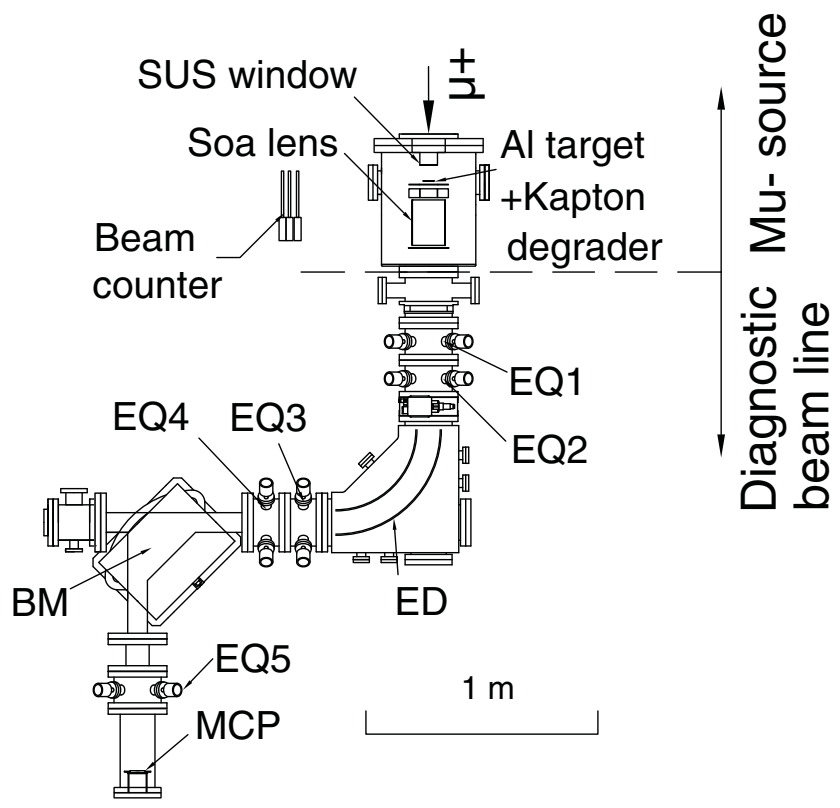

FIG. 1. Schematic drawing of an experimental setup of the $\mathrm{Mu}^{-}$ ion production experiment. A detailed description of the diagnostic beamline is given in the text.

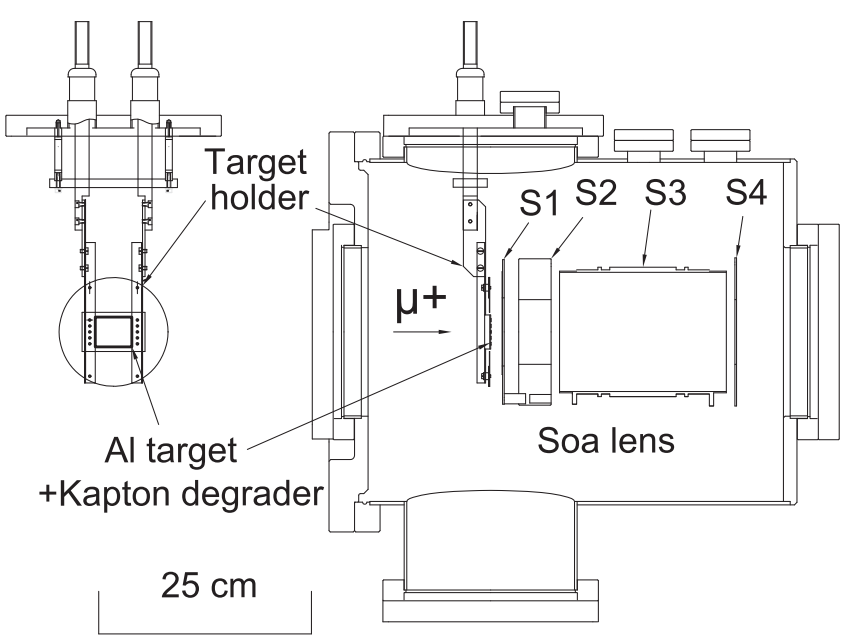

FIG. 2. Schematic drawing of the Al target and the Soa lens. 
the D-line and experimental apparatus, and a Kapton (Dupont inc.) degrader with a thickness of $150 \mu \mathrm{m}$ to decelerate the $\mu^{+}$'s. Then, the $\mu^{+}$'s were injected into the $\mathrm{Al}$ target with a purity of $99 \%$ and a thickness of $200 \mu \mathrm{m}$. The Kapton degrader was used for the fine adjustment of the stopping range of the $\mu^{+}$'s because the density of the Kapton is smaller than that of the Al target. Both the Kapton degrader and the Al target were rectangular with the dimension of $43 \times 35 \mathrm{~mm}^{2}$; the degrader was layered on the upstream side of the $\mathrm{Al}$ target, and both were mounted on the target holder. To measure the time at which the $\mu^{+}$'s entered the $\mathrm{Al}$ target, a beam counter composed of three plastic scintillation boards was positioned near the Al target. The dimensions of these boards, beginning with the one nearest to the $\mathrm{Al}$ target, were $3 \times 3.5 \times 0.5$, $6 \times 6.5 \times 0.5$, and $6 \times 5.5 \times 1 \mathrm{~mm}^{3}$, respectively.

The Soa lens consisted of five electrodes: Target, S1, S2, $\mathrm{S} 3$, and S4. The $\mathrm{Mu}^{-}$ions were accelerated to $20 \mathrm{keV}$ to be transported through a diagnostic beamline. For $20-\mathrm{keV}$ acceleration, the applied voltages of the Target, S1, S2, and S3 electrodes were 20.0, 18.9, 18.0, and $16.0 \mathrm{kV}$, respectively, while the S4 was grounded. The S1 was the mesh electrode, and its open aperture ratio was $85 \%$. The $\mathrm{Al}$ target and the Soa lens were the main components of the $\mathrm{Mu}^{-}$ion source. The RFQ was connected to the downstream of the Soa lens when the $\mathrm{Mu}^{-}$ions were accelerated with the RFQ.

The $\mathrm{Mu}^{-}$ions were transported using the diagnostic beamline and detected with a microchannel plate (MCP) located downstream of the diagnostic beamline. The diagnostic beamline consisted of one electrostatic deflector (ED), five electrostatic quadrupoles (EQs), and one bending magnet $(\mathrm{BM})$. The $\mathrm{Mu}^{-}$ion beam contained $\mu^{+}$'s that had penetrated the $\mathrm{Al}$ target, decay positrons from the $\mu^{+}$'s, and low-energy electrons produced by the field emission around the electrodes. These backgrounds should be distinguished from the $\mathrm{Mu}^{-}$ions using both kinetic energy and momentum information. They were suppressed by combining the ED and the BM. Voltages of $\pm 5 \mathrm{kV}$ were applied to each electrode to bend the $20-\mathrm{keV} \mathrm{Mu}^{-}$ion beam. The magnetic field of the BM was 250 Gauss with an applied current of $16.3 \mathrm{~A}$.

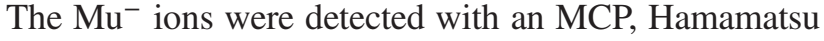
F2225-21P308 [22]. The diameter of the effective area was $40 \mathrm{~mm}$, and the open aperture ratio of the channels was $60 \%$. The MCP has a high sensitivity for low-energy muons $[23,24]$. The waveform from the MCP was recorded with a waveform digitizer, CAEN V1720 [25]. The hit timing and pulse height were extracted from the waveform. The $25-\mathrm{Hz}$ reference trigger from the J-PARC accelerator was used for the trigger of the digitizer. The incident $\mu^{+}$'s reached the $\mathrm{Al}$ target approximately $1 \mu \mathrm{s}$ after the start of the sampling.

The formation probability of the $\mathrm{Mu}^{-}$ions was determined by the measured event rate of $\mathrm{Mu}^{-}$ions. The relationship between the event rate of incident $\mu^{+}$'s at the just upstream of the chamber of the $\mathrm{Al}$ target, $R_{\text {inc } \mu^{+}}$, and the event rate of the $\mathrm{Mu}^{-}$ions measured with the MCP, $R_{\mathrm{Mu}^{-}}$, is described as

$$
R_{\mathrm{Mu}^{-}}=R_{\text {inc } \mu^{+}} \times \varepsilon_{\text {form }} \times \varepsilon_{\text {mesh }} \times \varepsilon_{\text {beam }} \times \varepsilon_{\mathrm{MCP}},
$$

where $\varepsilon_{\text {form }}$ is the formation probability of the $\mathrm{Mu}^{-}$ions, $\varepsilon_{\text {mesh }}$ is the transmission efficiency of the mesh electrode S1 in the Soa lens, $\varepsilon_{\text {beam }}$ is the beam transport efficiency through the Soa lens and the diagnostic beamline including the acceptance of the $\mathrm{Al}$ target, and $\varepsilon_{\mathrm{MCP}}$ is the detection efficiency of the MCP.

\section{SIMULATION}

A series of simulations were conducted to estimate the characteristics of the diagnostic beamline and the ratio of $\mu^{+}$'s irradiated on the Al target to the total number of incident $\mu^{+}$'s. First, the D-line was simulated with the G4beamline [26,27]. The initial distribution of the surface muon beam was generated at the downstream surface of the muon production target. The horizontal and vertical positions are defined as $x$ and $y$, respectively. The initial position distribution was calculated from the size of the primary proton beam injected into the muon production target. The momentum distribution was calculated from the relation of $d N_{\mu} / d p \propto p^{2.6}$ (See the Appendix) derived from the Bethe-Bloch formula [28], where $d N_{\mu}$ was the number of the muons per momentum $p$, assuming that the stopping distribution of the pion in the muon production target was uniform. The initial angular distribution was assumed to be isotropic because the surface muon was produced from the decay of the stopped pion. Figure 3 shows the simulated beam-profile distribution of the $\mu^{+}$'s transported to the $\mathrm{Al}$ target using the G4beamline.

Next, the beam transportation of the diagnostic beamline for the $\mathrm{Mu}^{-}$ions produced at the $\mathrm{Al}$ target was simulated.

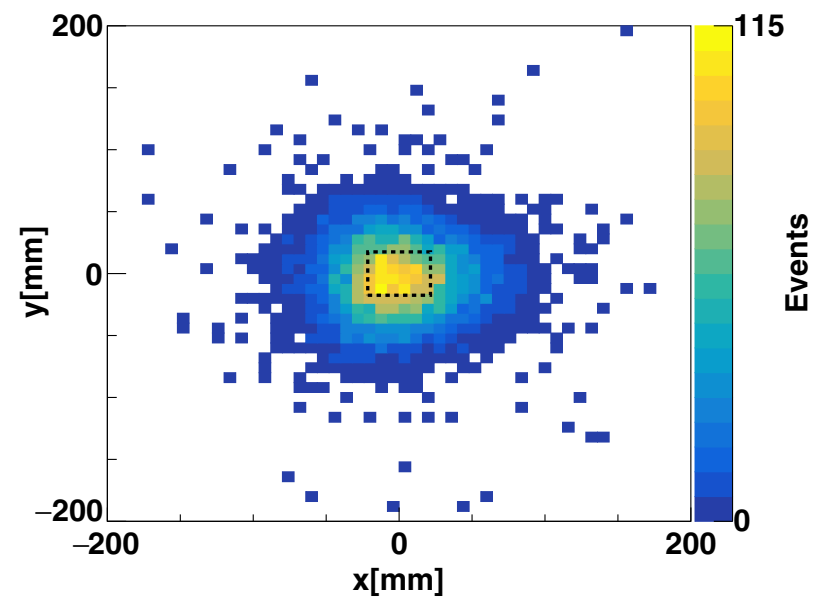

FIG. 3. Simulated beam-profile distribution of surface muons at the $\mathrm{Al}$ target. The dotted rectangle is a region of the $\mathrm{Al}$ target. 
First, the initial distribution of the $\mu^{+}$'s was generated at the upstream surface of the Kapton degrader. A twodimensional Gaussian distribution in the two transverse dimensions based on the measured beam size at this position was used. The energy loss and the multiple scattering in the Kapton degrader and the Al target were simulated using musrSim [29], a simulation program for $\mu \mathrm{SR}$ that can easily simulate the transportation of the muon beam. The energy (velocity) and angle distribution of the $\mu^{+}$'s at the downstream surface of the Al target, $f_{\mu^{+}}(v, \theta)$, was obtained, where $v$ and $\theta$ are the velocity and the angle of the $\mu^{+}$'s, respectively.

Assuming that the process of $\mathrm{Mu}^{-}$ion production is similar to the charge exchange process of the hydrogen atom [30], the energy and angle distribution of the $\mathrm{Mu}^{-}$ion, $f_{\mathrm{Mu}^{-}}(v, \theta)$, is described as

$$
f_{\mathrm{Mu}^{-}}(v, \theta)=f_{\mu^{+}}(v, \theta) Y_{-/+}(v),
$$

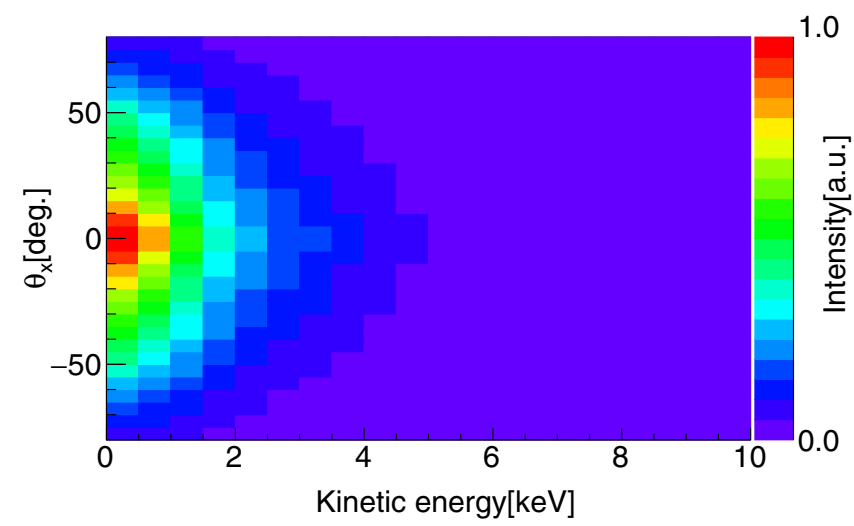

FIG. 4. Generated two-dimensional distribution for the $\mathrm{Mu}^{-}$ ions in terms of the kinetic energy and the emission angle. The number of generated events is $10^{6}$ events. The emission angle obtained by the horizontal direction of the momentum vector is drawn. The distribution in the vertical direction is similar.

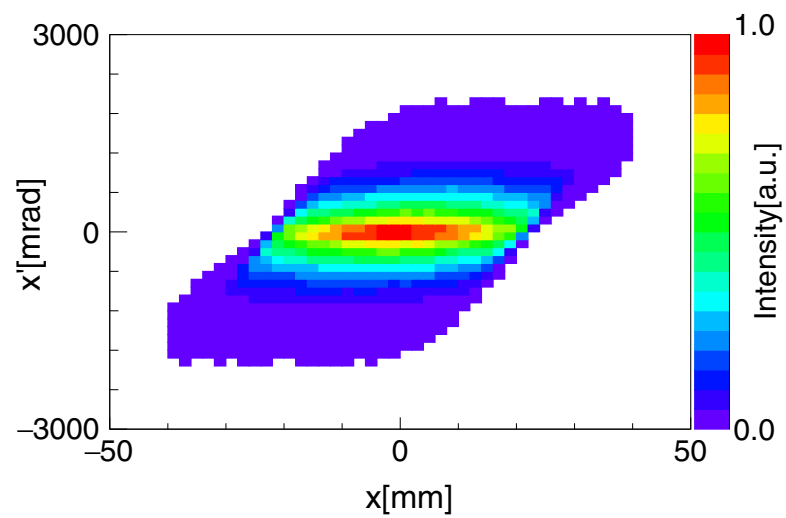

where $Y_{-/+}(v)$ is an experimental abundance ratio of the $\mathrm{H}^{-}$with respect to the $\mathrm{H}^{+}$from Ref. [30].

Figure 4 shows the two-dimensional distribution of the $\mathrm{Mu}^{-}$ions in terms of the kinetic energy and the emission angle using Eq. (2). This distribution was transported using musrSim. Electromagnetic field distributions of the Soa lens, the $\mathrm{ED}$, the $\mathrm{BM}$, and the EQs were calculated using OPERA [31] and implemented in musrSim. The beam transport efficiency of the $\mathrm{Mu}^{-}$ions through the Soa lens and the diagnostic beamline including the acceptance of the $\mathrm{Al}$ target, $\varepsilon_{\text {beam }}$, was evaluated based on the simulation, that was $2.3 \times 10^{-3}$. The decay loss of the $\mathrm{Mu}^{-}$ion was included in this efficiency. The dominant beam loss occurred around the Soa lens due to the large initial emittance of the $\mathrm{Mu}^{-}$ion beam. Normalized RMS horizontal and vertical emittance at the $\mathrm{Al}$ target were $25 \pi$ and $21 \pi \mathrm{mm} \mathrm{mrad}$, respectively, as shown in Fig. 5, while those at the exit of the Soa lens were $9.3 \pi$ and $8.4 \pi \mathrm{mm} \mathrm{mrad}$, respectively. The kinetic-energy acceptance of the diagnostic beamline was evaluated to be $1.4 \%$ (RMS).

Misalignment of the beamline components could be the major systematic uncertainty in the transmission efficiency. Thus, the effect of the possible case was examined. Each beamline component was confirmed to be aligned within $\pm 0.5 \mathrm{~mm}$ using the survey system during the assembly. The error of $\varepsilon_{\text {beam }}$ was evaluated using simulations where typical cases of misalignment were reproduced. Compared with the ideal situation, $\varepsilon_{\text {beam }}$ was reduced by $10 \%$ in the worst case, where all the components were assumed to be shifted to the same direction in the transverse plane and their effects of misalignment were piled up. Therefore, $\varepsilon_{\text {beam }}$ was evaluated as $\left(2.3_{-0.2}^{+0.0}(\right.$ syst $\left.)\right) \times 10^{-3}$.

\section{RESULTS}

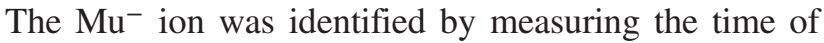
flight (ToF) and the pulse height using the MCP. The ToF is defined as the time difference between the hit timing of the

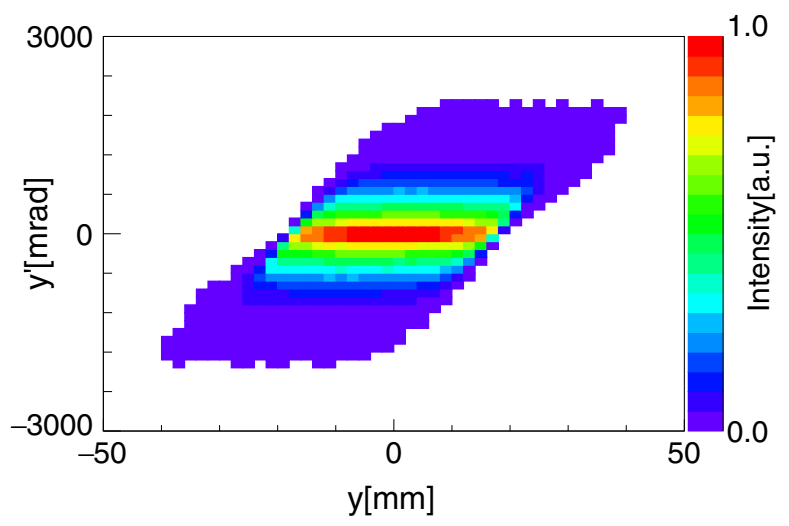

FIG. 5. Simulated phase-space distributions of the $\mathrm{Mu}^{-}$ion beam on the downstream surface of the Al target. The top and the bottom figures show the horizontal and vertical phase spaces, respectively. The number of generated events is $10^{6}$ events. The average kinetic energy of the $\mathrm{Mu}^{-}$ions was selected: $1 \mathrm{keV}(\beta \gamma=0.0044)$. 
MCP and the arrival time of the incident $\mu^{+}$'s at the Al target. As described in Sec. II, the arrival time was determined using the beam counter by detecting the positrons from the $\mu^{+}$'s decayed at the Al target. Timing calibration between the beam counter and the MCP was conducted using prompt positrons. Many positrons are generated at the muon production target together with the $\mu^{+}$'s. Passing through the D-line at the speed of light, these positrons arrive at the experimental area before the $\mu^{+}$'s; as such, they are called prompt positrons. Prompt positrons can be used as a time reference for the ToF because they are observed with both the beam counter and the MCP almost simultaneously.

Background events are suppressed using the pulse-height information of the MCP. The particles with opposite charges and different kinetic energies and momenta from the $\mathrm{Al}$ target can be suppressed with the ED and the BM. However, a lot of decay positrons from the $\mu^{+}$'s remained in the MCP signal because they could directly reach the MCP from anything of the beamline components, penetrating the vacuum chamber wall. By applying the pulseheight cut, $\mathrm{Mu}^{-}$-like events can be distinguished because the pulse height of the muon is higher than that of the decay positron $[23,24]$.

Figure 6 shows two-dimensional distribution of the ToF and pulse height for the extraction mode of negatively charged particles in the Soa lens and the diagnostic beamline. The signal region was defined as \pm 3 standard deviations (300 ns) of the pulse width of the incident $\mu^{+}$'s with respect to the expected arrival time of the $\mathrm{Mu}^{-}$ions at the MCP, while in the sideband region, the background events were dominant. The $\mu^{+}$'s decelerated through the $\mathrm{Al}$ target could be observed with the MCP in the positive extraction mode. The decelerated $\mu^{+}$'s were used to confirm the $\mathrm{ToF}$ of the $\mathrm{Mu}^{-}$ions because the energy of

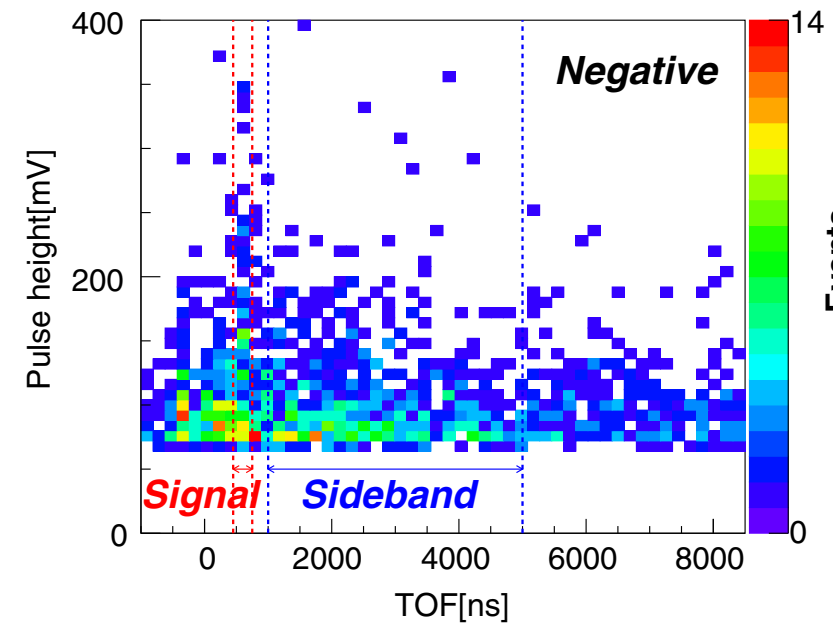

FIG. 6. Two-dimensional distribution of the ToF and the pulse height of the negative extraction. The number of incident $\mu^{+}$'s was $2.7 \times 10^{10}$. transported $\mu^{+}$'s was identical as that of the $\mathrm{Mu}^{-}$ions. Although decelerated $\mu^{+}$'s had a wide energy distribution, the diagnostic beamline was able to select the intended energy with limited energy acceptance. The mass of the $\mu^{+}$'s is nearly identical to that of the $\mathrm{Mu}^{-}$ions; thus, the ToF of the decelerated $\mu^{+}$'s is equal to that of the $\mathrm{Mu}^{-}$ions. The decelerated $\mu^{+}$'s were observed in the same signal region as the $\mathrm{Mu}^{-}$ions; therefore, the large-pulse-height events in the signal region of Fig. 6 were confirmed to be the $\mathrm{Mu}^{-}$ion events. Many $\mathrm{Mu}^{-}$-like events with large pulse heights were observed in the signal region of Fig. 6, while smaller pulse-height events were observed in the sideband region.

The net pulse-height distributions of the decelerated $\mu^{+}$'s and the $\mathrm{Mu}^{-}$ions were derived by subtracting the pulseheight distribution in the sideband region from that in the signal region of the two-dimensional distributions. Figure 7 shows the derived net-pulse-height distributions of the decelerated $\mu^{+}$'s and the $\mathrm{Mu}^{-}$ions. The pulse-height distributions of the $\mathrm{Mu}^{-}$ions and the decelerated $\mu^{+}$'s should be identical. The threshold of the pulse-height cut was set to $100 \mathrm{mV}$, which was low enough to have no effect on the formation probability of $\mathrm{Mu}^{-}$ions, as discussed below.

Figure 8 shows the ToF spectra of the slow $\mu^{+}$'s and $\mathrm{Mu}^{-}$ ions after the pulse-height cut was applied. Peaks around $300 \mathrm{~ns}$ were prompt positrons contaminated in the surface muon beam. The extended log-likelihood method [32] was used to evaluate the number of $\mathrm{Mu}^{-}$ions, and MINUIT [33] was used as the minimizer of the fitting. Probability density functions of the signal, $f_{\text {sig }}$, and the background, $f_{\text {bg }}$, were assumed to be the Gaussian and the exponential function, respectively, defined as follows:

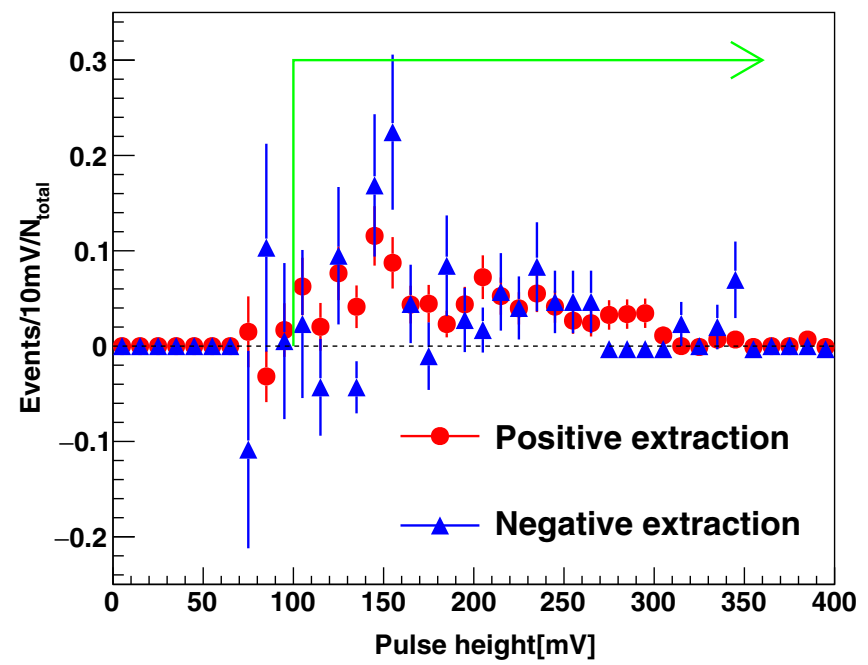

FIG. 7. Comparison of subtracted pulse-height distributions for decelerated $\mu^{+}$'s and $\mathrm{Mu}^{-}$ions. The histograms were normalized based on the number of total events. The green arrow indicates the threshold level applied to obtain ToF spectra shown in Fig. 8. 

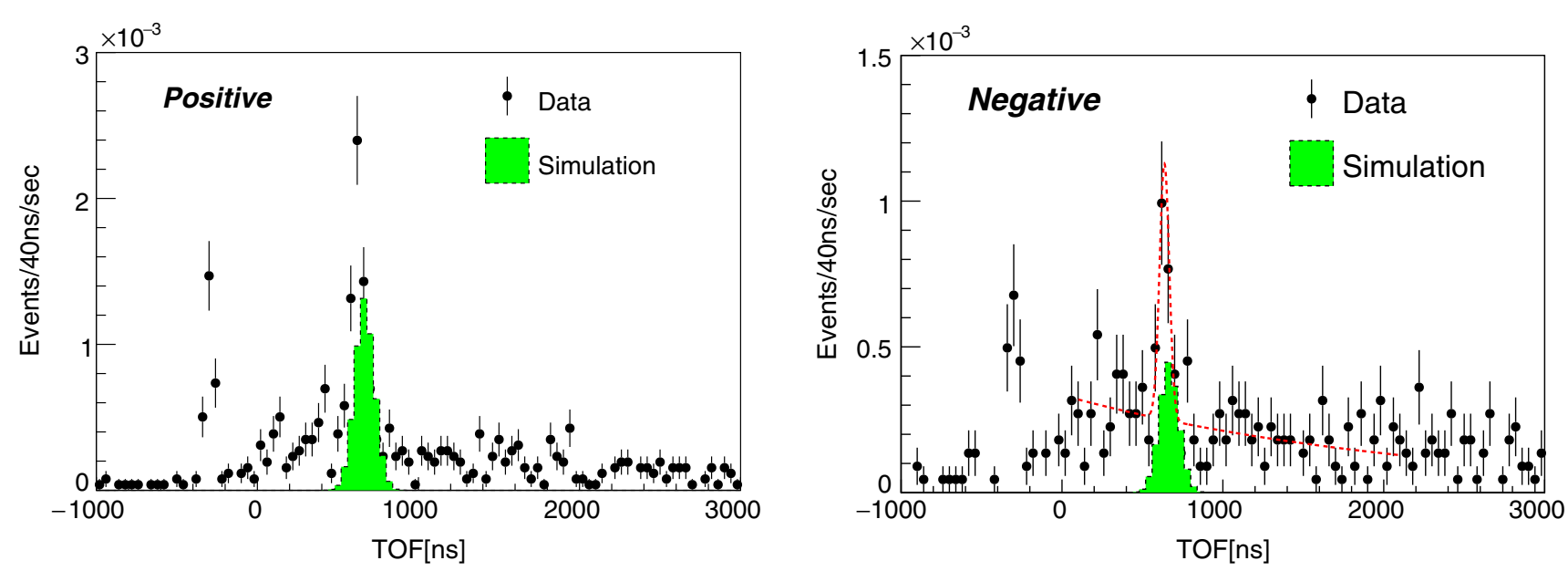

FIG. 8. The ToF spectra for the positive extraction (top) and negative extraction (bottom). The red dotted line in the negative extraction indicates the fitted function, and the green histograms indicate the simulated ToF distributions. Numbers of incident $\mu^{+}$'s for positive and negative extractions were $3.4 \times 10^{10}$ and $2.7 \times 10^{10}$, respectively. Peaks around $-300 \mathrm{~ns}$ were prompt positrons contaminated in the surface muon beam.

$$
\begin{gathered}
f_{\text {sig }}\left(t ; p_{\text {sig }}, a, \sigma\right)=\frac{p_{\text {sig }}}{\sqrt{2 \pi \sigma^{2}}} e^{\left(-\frac{(t-a)^{2}}{2 \sigma^{2}}\right)} \\
f_{\mathrm{bg}}\left(t ; p_{\mathrm{bg}}\right)=p_{\mathrm{bg}} C_{\mathrm{bg}} e^{\left(-\frac{t}{\tau}\right)}
\end{gathered}
$$

where $p_{\text {sig }}$ and $p_{\text {bg }}$ are the number of signal and background events, respectively; $a$ and $\sigma$ are the mean and standard deviation of the Gaussian in the signal function, respectively; $C_{\mathrm{bg}}$ is the normalization constant in the background function; and $\tau$ is the lifetime of the muon. The fitting parameters are $p_{\text {sig }}, p_{\text {bg }}, a$, and $\sigma$. The fitting results of the $\mathrm{Mu}^{-}$ions are shown in the bottom of Fig. 8 and indicated by the red dotted lines. The green histogram is the ToF distribution obtained by the simulation described in the previous section. The fitted ToF of the $\mathrm{Mu}^{-}$ions was $636.9 \pm 7.1 \mathrm{~ns}$, and the number of events for the $\mathrm{Mu}^{-}$ions was $36.6 \pm 7.5$. The number of beam pulses of incident $\mu^{+}$'s used for the analysis was $5.5 \times 10^{5}$ for the negative extraction. The total data taking time for the measurement was $2.2 \times 10^{4} \mathrm{~s}$ for the $\mathrm{Mu}^{-}$ions. Therefore, the measured event rate was $(1.7 \pm 0.3) \times 10^{-3} \mathrm{Mu}^{-} / \mathrm{s}$.

\section{DISCUSSION}

The formation probability of the $\mathrm{Mu}^{-}$ion is evaluated using Eq. (1). Table I shows the summary of the evaluation of the $\mathrm{Mu}^{-}$ion production. As shown in Sec. II, $R_{\text {inc } \mu^{+}}$was $1.3 \times 10^{6} \mu^{+} / \mathrm{s}$. Based on the simulation in Sec. III, $\varepsilon_{\text {beam }}$ was $\left(2.3_{-0.2}^{+0.0}\right) \times 10^{-3}$. The $\varepsilon_{\text {mesh }}$ was $8.5 \times 10^{-1}$, and $\varepsilon_{\mathrm{MCP}}$ was $6.0 \times 10^{-1}$ from their open aperture ratios. The $R_{\mathrm{Mu}^{-}}$ was $(1.7 \pm 0.3) \times 10^{-3} \mathrm{Mu}^{-} / \mathrm{s}$; therefore, $\varepsilon_{\text {form }}$ can be evaluated to be $\left(1.1 \pm 0.2(\text { stat })_{+0.1}^{-0.0}(\right.$ syst $\left.)\right) \times 10^{-6}$. The evaluated formation probability satisfied the requirement for the commissioning of the accelerator.

This developed $\mathrm{Mu}^{-}$ion source was successfully applied in a separate work of the muon acceleration experiment with the RFQ [34]. Applying the method of the evaluation in the total efficiency that was established in the present study, the efficiency in the acceleration experiment was determined to be $2.5 \times 10^{-4}$ with the beam losses in the Soa lens, RFQ, and the diagnostic beam line for the acceleration experiment but without the formation probability. The beam rate of incident $\mu^{+}$'s was $2.5 \times 10^{6} \mu^{+} / \mathrm{s}$ in the acceleration experiment; therefore, the expected event rate of accelerated $\mathrm{Mu}^{-}$ions was $\left(6.8 \pm 1.2(\text { stat })_{+0.4}^{-0.0}(\right.$ syst $\left.)\right) \times 10^{-4} \mathrm{Mu}^{-} / \mathrm{s}$, considering the formation probability from this result of the $\mathrm{Mu}^{-}$ion production experiment. Errors of the expected rate are predominantly originating from that in the formation probability determined by the present study. The measured event rate of the accelerated $\mathrm{Mu}^{-}$ions was $(5.1 \pm 0.8) \times 10^{-4} \mathrm{Mu}^{-} / \mathrm{s}$, so the measured event rate was consistent with the expected rate within the range of errors.

TABLE I. Summary of the evaluation of the $\mathrm{Mu}^{-}$ion production.

\begin{tabular}{lcc}
\hline \hline Term & Symbol & Value \\
\hline Event rate of $\mathrm{Mu}^{-}$ion & $R_{\mathrm{Mu}^{-}}$ & $\left(1.7 \pm 0.3^{\mathrm{a}}\right) \times 10^{-3}$ \\
Event rate of incident $\mu^{+}$ & $R_{\text {inc } \mu^{+}}$ & $1.3 \times 10^{6}$ \\
Form. prob. of $\mathrm{Mu}^{-}$ion & $\varepsilon_{\text {form }}$ & $\left(1.1 \pm 0.2^{\left.\mathrm{a}-0.0 \mathrm{~b}^{\mathrm{b}}\right) \times 10^{-6}}\right.$ \\
Trans. of mesh electrode & $\varepsilon_{\text {mesh }}$ & $8.5 \times 10^{-1}$ \\
Trans. of $\mathrm{Mu}^{-}$ion beam & $\varepsilon_{\text {beam }}$ & $\left(2.3_{-0.2}^{+0.0 \mathrm{~b}}\right) \times 10^{-3}$ \\
Detection eff. of $\mathrm{MCP}$ & $\varepsilon_{\mathrm{MCP}}$ & $6.0 \times 10^{-1}$ \\
\hline \hline
\end{tabular}

\footnotetext{
${ }^{\mathrm{a}}$ statistical error.

${ }^{\mathrm{b}}$ systematic error due to the misalignment.
} 


\section{CONCLUSION}

A $\mathrm{Mu}^{-}$ion source using an $\mathrm{Al}$ target was developed as a low-energy muon source. This $\mathrm{Mu}^{-}$ion source can directly produce a cooled $\mathrm{Mu}^{-}$ion beam from the surface muon beam using a simple apparatus essentially composed of an $\mathrm{Al}$ foil only. The $\mathrm{Mu}^{-}$ion emission from the metal surface was studied by the precursor study [2], and this phenomenon was first applied to the ion source for the accelerator. In the $\mathrm{Mu}^{-}$ion production experiment, the measured event rate and the formation probability of the $\mathrm{Mu}^{-}$ions were $(1.7 \pm 0.3) \times 10^{-3} \mathrm{Mu}^{-} / \mathrm{s}$ and $\left(1.1 \pm 0.2(\mathrm{stat})_{+0.1}^{-0.0}(\mathrm{syst})\right) \times 10^{-6}$, respectively. Using this $\mathrm{Mu}^{-}$ion source, the accelerated $\mathrm{Mu}^{-}$ions were successfully observed in the first demonstration of the muon acceleration with the RFQ [34]. The measured event rate of the accelerated $\mathrm{Mu}^{-}$ions was consistent with that of the simulation. This $\mathrm{Mu}^{-}$ion source is an essential and practical device for the development of a muon accelerator, and its reliability was experimentally confirmed by the actual commissioning of the accelerator.

\section{ACKNOWLEDGMENTS}

We would like to deeply thank the RIKEN Advanced Meson Science Laboratory and the J-PARC MUSE group for their significant supports. This work is supported by JSPS KAKENHI Grants No. JP25800164, No. JP15H03666, No. JP16H03987, No. JP15H05742, No. JP16J07784, and No. JP18H03707. This work is also supported by the Korean National Research Foundation Grants No. NRF2015H1A2A1030275, No. NRF-2015K2A2A4000092, and No. NRF-2017R1A2B3007018; the Russian Foundation for Basic Research Grant No. RFBR 17- 52-50064; and the Russian Science Foundation Grant No. RNF 17-12-01036. The muon experiment at the Materials and Life Science Experimental Facility of the J-PARC was performed under user programs (Proposal No. 2016A0067).

\section{APPENDIX: CALCULATION OF SURFACE-MUON-MOMENTUM DISTRIBUTION}

The surface muons are emitted from pions stopping near the surface of the muon production target, and their momentum distribution is known to be expressed by a power function of the momentum [35]. The cutoff of the muon momentum distribution has a maximum edge of $29.8 \mathrm{MeV} / c$. The momentum distribution reflects the energy loss of the muon in the muon production target, and thus is approximated by the 2.6th power of the momentum. We derive the momentum distribution of the surface muon from the generic Bethe-Bloch formula [28], which is described as

$$
-\frac{d E}{d x}=\frac{G}{\beta^{2}} \ln \left(\frac{2 m_{e} c^{2} \beta^{2}}{I}\right)
$$

where $G \equiv 4 \pi e^{4} N Z / m_{e} c^{2} ; e$ is the charge of the muon; $m_{e}$ is the mass of the electron; $N$ and $Z$ are the density and the atomic number of the absorber, respectively; $\beta$ is defined as $\beta=v / c$, where $v$ is the velocity of the muon, and $c$ is the speed of light; and $I \approx 10 Z(\mathrm{eV})$ is the ionization energy of the absorber. The relation between the energy $E$ and the momentum $p$ of the muon is $E=\sqrt{\left(m_{\mu} c^{2}\right)^{2}+(p c)^{2}}$, where $m_{\mu}$ is the mass of the muon. Thus, the energy deposit is related to momentum loss:

$$
-\frac{d E}{d x}=\frac{p c}{E}\left(\frac{d p}{d x}\right)
$$

Substituting Eq. (A2) into Eq. (A1), $d E / d x$ can be converted to $d p / d x$ as

$$
\frac{d p}{d x}=-\frac{G}{\beta^{3}} \ln \left(\frac{2 m_{e} c^{2}}{I} \beta^{2}\right) .
$$

The logarithm function in Eq. (A3) is approximated by the power function. The Maclaurin expansion centered on $\beta=\beta_{0}$ for $f(\beta) \equiv \ln \left(2 m_{e} c^{2} \beta^{2} / I\right)$ is

$$
\begin{aligned}
f(\beta) & =\ln \left[\frac{2 m_{e} c^{2} \beta_{0}^{2}}{I}(1+h)^{2}\right] \\
& =C+2 \ln (1+h) \\
& =C+2\left(h-\frac{h^{2}}{2}+\cdots\right) \\
& =C\left(1+\frac{2}{C} h-\frac{1}{C} h^{2}+\cdots\right),
\end{aligned}
$$

where $h \ll 1, \beta=\beta_{0}(1+h)$ and $C=\ln \left(2 m_{e} c^{2} \beta_{0}^{2} / I\right)$. When the approximate function of $f(\beta)$ is $g(\beta) \equiv A \beta^{\alpha}$, where $\alpha$ is the real number and $A$ is an arbitrary constant, the Maclaurin expansion of $g(\beta)$ is

$$
\begin{aligned}
g(\beta) & =A \beta_{0}^{\alpha}(1+h)^{\alpha} \\
& =A \beta_{0}^{\alpha}\left[1+\alpha h+\frac{\alpha(\alpha-1)}{2 !} h^{2}+\cdots\right] .
\end{aligned}
$$

Higher-order terms in Eq. (A4) and (A5) are negligible due to $h \ll 1$. Thus, in case of $f(\beta) \approx g(\beta)$, based on a comparison between coefficients of first-order terms in Eq. (A4) and (A5), $\alpha$ is calculated as

$$
\alpha=\frac{2}{C}=2\left[\ln \left(\frac{2 m_{e} c^{2} \beta_{0}^{2}}{I}\right)\right]^{-1} .
$$

In case of the surface muon, $\beta \sim 0.25, \alpha$ is calculated as $0.3 \sim 0.4$; thus, $d p / d x$ is typically described as 


$$
\frac{d p}{d x} \propto p^{-2.6}
$$

The number of the surface muons per momentum, $\Delta N / \Delta p$, is $\Delta N / \Delta p=(\Delta N / \Delta x)(\Delta p / \Delta x)^{-1}$, where $\Delta N / \Delta x$ is the number of the muons at a certain region $\Delta x$, assuming the stopping distribution of the pion in the muon production target is uniform. Finally, the momentum distribution of the surface muon is calculated as the function of

$$
\frac{d N}{d p} \propto p^{2.6}
$$

[1] D. R. Harshman, J. B. Warren, J. L. Beveridge, K. R. Kendall, R. F. Kiefl, C. J. Oram, A. P. Mills, Jr., W. S. Crane, A. S. Rupaal, and J. H. Turner, Observation of LowEnergy $\mu^{+}$Emission from Solid Surfaces, Phys. Rev. Lett. 56, 2850 (1986).

[2] Y. Kuang, A.-P. Arnold, F. Chmely, M. Echhause, V. W. Hughes, J. R. Kane, S. Kettell, D.-H. Kim, K. Kumar, D. C. Lu, B. Matthias, B. Ni, H. Orth, G. zu Putlitz, H. R. Schaefer, P. A. Souder, and K. Woodle, Formation of the negative muonium ion and charge-exchange processes for positive muons passing through thin metal foils, Phys. Rev. A 39, 6109 (1989).

[3] R. Kitamura, M. Otani, Y. Fukao, N. Kawamura, T. Mibe, Y. Miyake, K. Shimomura, Y. Kondo, K. Hasegawa, S. Bae, B. Kim, G. Razuvaev, H. Iinuma, K. Ishida, and N. Saito, First trial of the muon acceleration for J-PARC muon g-2/EDM experiment, J. Phys. Conf. Ser. 874, 012055 (2017).

[4] F. Scheck, Muon physics, Phys. Rep. 44, 236 (1978).

[5] M. Abe et al., A new approach for measuring the muon anomalous magnetic moment and electric dipole moment, Prog. Theor. Exp. Phys. 2019, 053 C02 (2019).

[6] Y. Miyake et al., Ultra slow muon project at J-PARC MUSE, J. Phys. Soc. Jpn. Conf. Proc. 2, 010101 (2014).

[7] W. Higemoto et al., Materials and life science experimental facility at the Japan proton accelerator research complex IV: The muon facility, Quantum Beam Sci. 1, 11 (2017).

[8] K. Nagamine, Y. Miyake, K. Shimomura, P. Birrer, J. P. Marangos, M. Iwasaki, P. Strasser, and T. Kuga, Ultraslow Positive-Muon Generation by Laser Ionization of Thermal Muonium from Hot Tungsten at Primary Proton Beam, Phys. Rev. Lett. 74, 4811 (1995).

[9] P. Bakule et al., Measurement of muonium emission from silica aerogel, Prog. Theor. Exp. Phys. 2013, $103 \mathrm{C} 01$ (2013).

[10] G. A. Beer et al., Enhancement of muonium emission rate from silica aerogel with a laser ablated surface, Prog. Theor. Exp. Phys. 2014, 091C01 (2014).

[11] T. Prokscha, E. Morenzoni, C. David, A. Hofer, H. Glückler, and L. Scandella, Moderator gratings for the generation of epithermal positive muons, Appl. Surf. Sci. 172, 235 (2001).

[12] E. Morenzoni et al., Low-energy $\mu \mathrm{SR}$ at PSI: present and future, Physica B (Amsterdam) 289-290, 653 (2000).
[13] D. R. Harshman, A. P. Mills, Jr., J. L. Beveridge, K. R. Kendall, G. D. Morris, M. Senba, J. B. Warren, A. S. Rupaal, and J.H. Turner, Generation of slow positive muons from solid rare-gas moderators, Phys. Rev. B 36, 8850 (1987).

[14] Y. Kondo et al., Re-Acceleration of Ultra Cold Muon in J-PARC Muon Facility, in Proc. of IPAC18, Vancouver, Canada (JACoW, Geneva, 2018), FRXGBF1.

[15] M. Otani, N. Kawamura, T. Mibe, T. Yamazaki, K. Ishida, and G. Marshal, Simulation of surface muon beamline, ultraslow muon production and extraction for the J-PARC g-2/EDM experiment, J. Phys. Conf. Ser. 1067, 052018 (2018).

[16] Y. Kondo et al., Simulation study of muon acceleration using RFQ for a new muon g-2 experiment at J-PARC, in Proc. of IPAC2015, Richmond, VA, USA, 2015 (JACoW, Geneva, 2015), TPHF045, pp. 3801-3803.

[17] M. Otani, T. Mibe, M. Yoshida, K. Hasegawa, Y. Kondo, N. Hayashizaki, Y. Iwashita, Y. Iwata, R. Kitamura, and N. Saito, Interdigital H-mode drift-tube linac design with alternative phase focusing for muon linac, Phys. Rev. Accel. Beams 19, 040101 (2016).

[18] M. Otani et al., Disk and washer coupled cavity linac design and cold-model for muon linac, J. Phys.: Conf. Ser. 1350, 012097 (2019).

[19] Y. Kondo et al., High-beta section of a muon linac for the measurement of the muon g-2/EDM at J-PARC, in Proc. of the 14th Annual Meeting of PASJ, Sapporo, Japan (Particle Accelerator Society of Japan, Sapporo, 2017), TUP036, pp. 398-400, https:/www.pasj.jp/web_publish/pasj2017/ proceedings/PDF/TUP0/TUP036.pdf.

[20] Y. Kondo, T. Morishita, K. Hasegawa, E. Chishiro, K. Hirano, T. Hori, H. Oguri, F. Sato, and S. Shionozaki, High-power test and thermal characteristics of a new radiofrequency quadrupole cavity for the Japan Proton Accelerator Research Complex linac, Phys. Rev. Accel. Beams 16, 040102 (2013).

[21] K. F. Canter, P. H. Lippel, W. S. Crane, and A. P. Mills, Jr., Positron Studies of Solids, Surfaces and Atoms (World Scientific, Singapore, 1986), p. 199.

[22] "MCP (Microchannel plate) and MCP assembly", TMCP0002E05 (HAMAMATSU Photonics Co., Shizuoka, Japan, 2016).

[23] B. Kim et al., Development of a microchannel plate based beam profile monitor for a re-accelerated muon beam, Nucl. Instrum. Methods Phys. Res., Sect. A 899, 22 (2018).

[24] M. Otani et al., Response of microchannel plates to positrons from muon-decays, Nucl. Instrum. Methods Phys. Res., Sect. A 943, 162475 (2019).

[25] CAEN Mod. V17208 CHANNEL 12 BIT $250 \mathrm{MS} / \mathrm{S}$ DIGITIZER technical information manual, revision no. 26 (2014).

[26] Geant4 beamline, http://public.muonsinc.com/Projects/ G4beamline.aspx.

[27] Geant4, http://geant4.cern.ch/.

[28] G. Knoll, Radiation Detection and Measurement, 4th ed. (John Wiley \& Sons, New York, 1989).

[29] musrSim, https://www.psi.ch/lmu/geant4-simulations. 
[30] M. Gonin, R. Kallenbach, and P. Bochsler, Charge exchange of hydrogen atoms in carbon foils at $0.4-120 \mathrm{keV}$, Rev. Sci. Instrum. 65, 648 (1994).

[31] OPERA 3D Software for electro-magnetic design by Vector Field.

[32] G. Cowan, Statistical Data Analysis (Oxford University Press, New York, 1998).

[33] F. James, MINUIT function minimization and error analysisreference manual, version 94.1, CERN Program Library
Long Writeup D506, https://root.cern.ch/download/minuit .pdf.

[34] S. Bae et al., First muon acceleration using a radiofrequency accelerator, Phys. Rev. Accel. Beams 21, 050101 (2018).

[35] A. E. Pifer, T. Bowen, and K. R. Kendall, A high stopping density $\mu^{+}$beam, Nucl. Instrum. Methods $\mathbf{1 3 5}$, 39 (1976). 